\title{
Caracterización fenotípica y genotípica de Ornithobacterium rhinotracheale procedentes de aves de corral con cuadros clíni- cos respiratorios en el Perú entre 2015 y 2017
}

\author{
Phenotypic and genotypic characterization of Ornithobacterium rhinotracheale \\ from poultry with respiratory clinical signs in Peru between 2015 and 2017
}
Rony Yunior Cotaquispe Nalvarte ${ }^{1}$, Jorge Rodríguez Bailón ${ }^{1,2}$, Ysabel Koga Yanagui ${ }^{1}$, Robert Tinoco Romero', Irene Delgado De La Flor Montaubán², Alberto Manchego Sayán ${ }^{3}$

\section{Resumen}

\begin{abstract}
Ornithobacterium rhinotracheale, al igual que otras bacterias asociadas a cuadros de orden respiratorio, viene siendo sometida a una exigente presión de selección debido al uso de desinfectantes y antibióticos, lo cual ha venido generando cepas multidrogo resistentes, así como un incremento en la variabilidad genética. El objetivo del estudio fue caracterizar 36 aislados de $O$. rhinotracheale provenientes de aves de corral de cinco regiones del Perú, mediante un perfil de ocho antibióticos (fosfomicina, amoxicilina, ciprofloxacina, enrofloxacina, norfloxacina, sulfatrimetopim, doxiciclina, oxitetraciclina), y genotipificación por técnicas de ERIC-PCR. Los resultados indican la presencia de multidrogo resistencia de 2 a 6 antibióticos en $94.4 \%(n=34)$ de las cepas y el 100\% resistente al menos a una familia de antibióticos. La resistencia más común fue a la fosfomicina (77.8\%) seguido del sulfatrimetopim (75.0\%). Se encontraron 11 perfiles genéticos diferenciados (A-K), siendo el perfil D más frecuente en los aislados (55.6\%). Los resultados sugieren la presencia de cepas de $O$. rhinotracheale con moderada variabilidad y perfiles de resistencia a los antibiótico fosfomicina y sulfatrimetopim.
\end{abstract}

Palabras clave: Ornithobacterium rhinotracheale; resistencia a antibióticos; genotipificación; ERIC-PCR

\footnotetext{
${ }^{1}$ Laboratorio Bioservice SRL, Lima, Perú

${ }^{2}$ Unidad de Biotecnología Molecular, Laboratorios de Investigación y Desarrollo (LID), Facultad de Ciencias y Filosofia, Universidad Peruana Cayetano Heredia, Lima, Perú

${ }^{3}$ Laboratorio de Microbiología y Parasitología Veterinaria, Facultad de Medicina Veterinaria, Universidad Nacional Mayor de San Marcos, Lima, Perú

${ }^{4}$ E-mail:r.cotaquispe.n@gmail.com
}

Recibido: 25 de febrero de 2019

Aceptado para publicación: 23 de octubre de 2019 
Ornithobacterium rhinotracheale, like other bacteria associated with respiratory conditions, has been subjected to a demanding selection pressure due to the use of disinfectants and antibiotics, which has been generating resistant multidrug strains, as well as an increase in its genetic variability. The objective of the study was to characterize 36 O. rhinotracheale isolates from poultry from five regions of Peru by a profile of eight antibiotics (fosfomycin, amoxicillin, ciprofloxacin, enrofloxacin, norfloxacin, sulfatrimetropin, doxycycline, oxytetracycline), and genotyping by ERIC-PCR techniques. The results indicate the presence of multidrug resistance of 2 to 6 antibiotics in $94.4 \%$ (n $=34$ ) of the strains and $100 \%$ were resistant to al least one antibiotic family. The most common resistance was to fosfomycin (77.8\%) and sulfatrimetropin (75.0\%). At least, 11 differentiated genetic profiles (A-K) were found, being the most frequent the D profile in the isolates $(55.6 \%)$. The results suggest the presence of $O$. rhinotracheale strains with moderate variability and antibiotic resistance profiles fosfomycin and sulfatrimetropin.

Key words: Ornithobacterium rhinotracheale; antibiotic resistance; genotyping; ERICPCR

\section{INTRODUCCIÓN}

\section{Ornithobacterium rhinotracheale} (ORT) es un bacilo gramnegativo pleomórfico, de colonias convexas, lisas, no hemolíticas (van Empel y Hafez, 1999), presente en el tracto respiratorio de varias especies de aves domésticas y silvestres (Vandamme et al., 1994; Hafez y Sting, 2011). La infección por $O$. rhinotracheale se caracteriza principalmente por presentar signos respiratorios, disnea, estornudos, disminución en la producción de huevos; así mismo, en asociación con otros patógenos produce un aumento considerable de la mortalidad, conllevando a considerables pérdidas económicas (Macagnan, 2006).

Se han reportado varios casos de infecciones respiratorias asociados a esta bacteria en Perú (Hung y Alvarado, 2001), Japón (Sakai et al., 2000), Turquía (Turan, 2002), Canadá (Joubert et al., 1999), Estados Unidos (Amonsin et al., 1997), Israel (El-Sukhon et al., 2002) y México (Soriano et al., 2002).
Actualmente se han aislado 18 serotipos nombrados de A hasta la R (Ha et al., 2016; Turan y Ak, 2002). Algunos de estos serovares (A, $\mathrm{B}, \mathrm{D}, \mathrm{E})$ producen reacciones cruzadas a nivel serológico, dando indicios sobre la existencia de subespecies de $O$. rhinotrache (Hafezy Vandamme, 2011; Macagnan, 2006).

La aplicación de la reacción en cadena de la polimerasa (PCR) para la detección molecular de $O$. rhinotracheale en el Perú ha sido eficiente, rápida y confiable (Hung y Alvarado, 2001), siendo útil, además, para detectar la diversidad genética de estos aislados (Koga y Zavaleta, 2005). Métodos de tipificación molecular basados en PCR (ERIC-PCR) han dado buenos resultados como modelo para tipificación bacteriana (Vargas et al., 2003). La técnica de ERICPCR se basa en la amplificación de regiones intergénicas palindromicas consecutivas dentro del cromosoma bacteriano, permitiendo incluso discriminar líneas clonales mutantes circulantes a nivel de bacterias (Espinoza et al., 2011; Peña et al., 2016; Rèka et al., 2017). 
El incremento de la exposición de microorganismos a sustancias desinfectantes o antimicrobianas ha generado un proceso adaptativo en las bacterias debido a un incremento en la presión de selección (Chou et al., 2009). Cambios en el medio ambiente donde se desarrollan las bacterias requiere un incremento en variabilidad genética intra e interespecífica que permitan la aparición y posterior selección de variantes genéticas con mayor adaptación y patogenicidad (Soriano et al., 2003; Macagnan, 2006).

En el Perú, no existe suficiente información sobre tipificación molecular que permitan determinar la variabilidad genética y la potencial presencia de variantes genéticas de $O$. rhinotracheale, siendo de utilidad para estudios epidemiológicos y la aplicación de estrategias de prevención y control. El objetivo del presente estudio fue caracterizar cepas circulantes de $O$. rhinotracheale procedentes de cinco regiones del Perú, mediante técnicas de tipificación molecular ERIC-PCR y perfiles de resistencia antimicrobiana.

\section{Materiales y Métodos}

Treinta y seis cepas de Ornithobacterium rhinotracheale fueron aisladas de senos infraorbitarios, cornetes nasales, tráquea y sacos aéreos de aves afectadas con sintomatología y lesiones compatibles con infección por $O$. rhinotracheale, provenientes de los departamentos de Lima, Ica, Arequipa, La Libertad y Ucayali (Perú) entre 2015 y 2017.

Se determinó el perfil de resistencia antimicrobiana para ocho antibióticos de importancia veterinaria distribuidos en cinco familias (sulfonamidas potenciadas: sulfatrimetopim; quinolonas: norfloxacina, enrofloxacina, ciprofloxacina; tetraciclinas: oxitetraciclina, doxiciclina; penicilinas: amoxicilina; y fosfonatos: fosfomicina). El perfil fue determinado mediante el método de difusión en agar Mueller Hinton-sangre al 5\%, descrito por el Comité Nacional de Estándares Clínicos de Laboratorio (NCCLS, 2017).

El ADN genómico de las 36 cepas fue extraído mediante el método de membranas de sílica gel utilizando el kit de extracción de ADN GF-1 Tissue DNA Extraction (Vivantis), siguiendo las indicaciones del fabricante. El ADN genómico fue almacenado a $-20{ }^{\circ} \mathrm{C}$ hasta su procesamiento.

$O$. rhinotracheale fue detectada mediante amplificación por PCR específica de un fragmento de 784 pares de bases pertenecientes al gen 16S rRNA utilizando los cebadores descritos por Espinoza et al. (2017): OR16S - F1 (5'-GAGAATTAATTTACGGATTAAG-3') y OR16S-R1 (5'-TTCGCTTGGT CTCCGAAGAT-3').

La reacción de PCR se realizó en un volumen final de $20 \mu 1$ conteniendo $10 \mathrm{X} \mathrm{Bu}$ ffer PCR, $0.25 \mathrm{mM}$ de dNTPs, $2.5 \mathrm{mM}$ de $\mathrm{MgCl}_{2}, 10$ pmol de cada cebador, $1 \mathrm{U}$ Taq DNA polimerasa (Fermentas) y $30 \mathrm{ng}$ de ADN genómico. Los ciclos termales fueron los siguientes: 1 ciclo de desnaturalización inicial PCR de $95{ }^{\circ} \mathrm{C}$ por $4 \mathrm{~min} ; 35$ ciclos de PCR: desnaturalización de $94{ }^{\circ} \mathrm{C}$ por $30 \mathrm{~s}$, alineamiento de $58^{\circ} \mathrm{C}$ por $60 \mathrm{~s}$ y extensión de $72{ }^{\circ} \mathrm{C}$ por $90 \mathrm{~s}$; 1 ciclo de extensión final de PCR de $72{ }^{\circ} \mathrm{C}$ por $5 \mathrm{~min}$. La amplificación se llevó a cabo en un termociclador Veriti Thermal Cycler (Life Technologies).

Los productos de PCR fueron separados mediante electroforesis en gel de agarosa al $1 \%$ en buffer TBE $1 \mathrm{X}$ (Tris $0.89 \mathrm{M}$, Borato $0.02 \mathrm{M}$, EDTA $0.89 \mathrm{M}, \mathrm{pH}$ 8.3) durante 45 min a $100 \mathrm{v}$ y visualizados bajo luz UV. Adicionalmente, los productos de PCR fueron secuenciados por ambas hebras utilizando BigDye Terminator v3.1 Cycle Sequencing Kit (Thermo Scientific) utilizando un analizador genético ABI 3137 XL (Life Technologies). El servicio de secuenciamiento fue proveído por la empresa Macrogen Inc. 


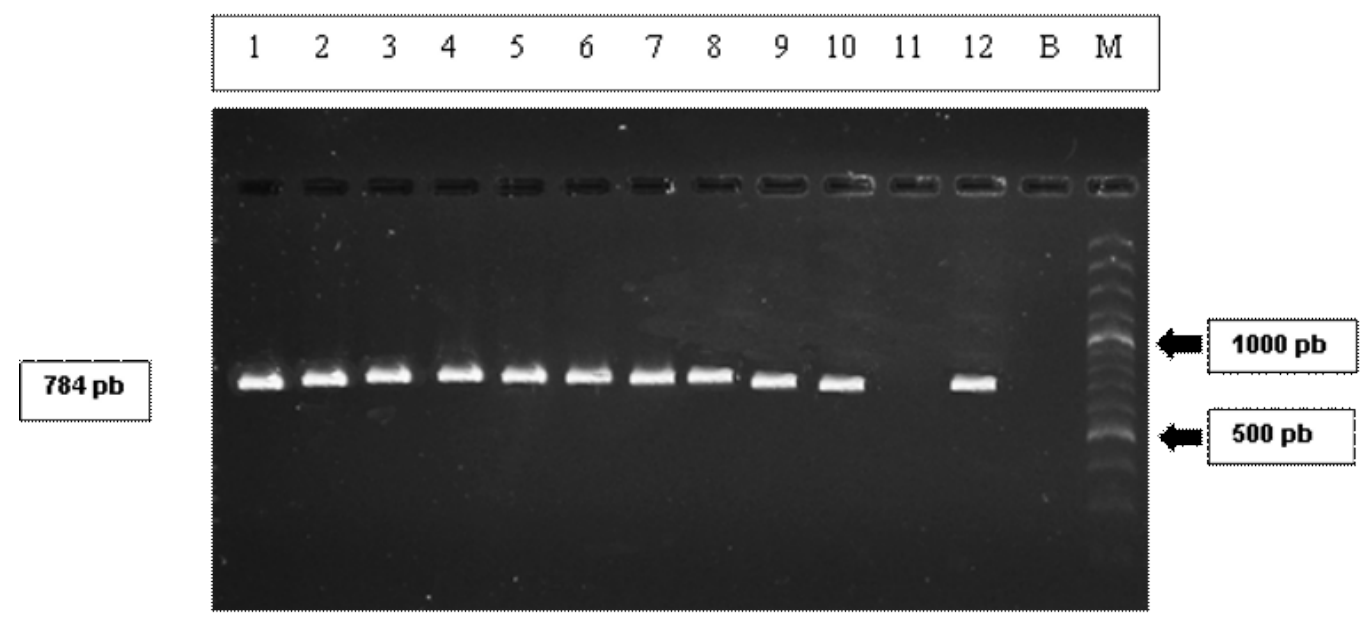

Figura 1. Amplificación específica para detección de Ornithobacterium rhinotracheale por PCR del gen 16S rRNA. Carril 1 a 10: ADN obtenido de cepa de $O$. rhinotracheale; Carril 11. Control negativo, ADN de Pasteurella multicida ATCC® 12945; Carril 12. Control positivo, ADN de O. rhinotracheale ATCC® 51464, Carril B: Blanco de PCR; Carril M, Marcador de peso molecular GeneRuler 100 bp Plus DNA Ladder (Thermo Scientific)

Cuadro 1. Perfiles de resistencia a ocho antibióticos en 36 cepas aisladas de Ornithobacterium rhinotracheale en aves comerciales del Perú con infecciones respiratorias.

\begin{tabular}{lccc}
\hline Antimicrobiano & Resistentes (\%) & Intermedias (\%) & Sensibles (\%) \\
\hline Fosfomicina & 77.8 & 2.8 & 19.4 \\
Amoxicilina & 36.1 & 5.6 & 58.3 \\
Ciprofloxacina & 41.7 & 19.4 & 38.9 \\
Enrofloxacina & 52.8 & 5.6 & 41.7 \\
Doxiciclina & 58.3 & 27.8 & 13.9 \\
Norfloxacina & 63.9 & 11.1 & 25.0 \\
Sulfatrimetopim & 75.0 & 5.6 & 19.4 \\
Oxitetraciclina & 58.3 & 8.3 & 33.3 \\
\hline
\end{tabular}

El perfil genético (ADN fingerprinting) fue realizado a partir de los patrones de bandas generadas por ERIC-PCR utilizando el programa GelAnalyzer 2010 (Peña et al., 2016). Los patrones de bandeo de ERICPCR fueron transformados a una matriz de (dis)similaridad utilizando el coeficiente de Jaccard y Dice (Mendoza et al., 2014) mediante el programa FAMD v1.25 (Schluter y
Harris, 2006). El análisis de la variabilidad genética intra e interespecífica para las 36 cepas de $O$. rhinotracheale fue realizado utilizando el método de máxima similaridad del análisis de varianza molecular (AMOVA) y dendogramas generados mediante el método Neighbor Joining en base a una matriz de distancia genética con los programas FAMD v. 1.25 (Schlüter, 2006) y Mega v. 6.0 (Tamura et al., 2013). 
Resultados

El total de los aislados $(n=36)$ presentaron un patrón morfológico en agar MuellerHinton-sangre al $5 \%$ y perfil bioquímico compatible con $O$. rhinotracheale. Así mismo, la totalidad de aislados fue confirmada como $O$. rhinotracheale mediante amplificación y secuenciamiento de un fragmento de PCR del gen ribosomal 16S rRNA (Figura 1).

Los aislados mostraron una elevada resistencia antimicrobiana, siendo el $100 \%$ resistente al menos a una familia de antibióticos y el $94.4 \%$ fueron multidrogo resistentes (resistencia al menos a dos familias de antibióticos). Los aislados mostraron perfiles de resistencia principalmente para fosfomicina $(77.8 \%)$, sulfatrimetopim $(75.0 \%)$ y norfloxacina $(63.9 \%)$, mientras que la mayor sensibilidad fue para la amoxicilina (58.3\%) (Cuadro 1).

Se observaron siete bandas polimórficas (loci) comprendidas entre 200 y 2000 pares de bases con un total de 11 perfiles de ERICPCR identificados (A-K), siendo el más abundante el perfil D con $55.6 \%(20 / 36)$ de los aislados, seguido del perfil $\mathrm{F}(11.1 \%, 4 / 36)$ (Figura 2). Se apreció una moderada diferenciación entre las cepas de $O$. rhinotracheale, las cuales se agruparon en al menos dos clústeres genéticos sin correspondencia con el lugar de origen de los aislados, sugiriendo la posibilidad de un origen común dentro de las cepas provenientes de las cinco regiones del país. La región Lima presentó la mayor variabilidad genética con la presencia 6 de 11 patrones genéticos, de los cuales cinco fueron patrones únicos $(\mathrm{B}, \mathrm{E}, \mathrm{F}, \mathrm{J}, \mathrm{K})$ (Cuadro 2).

\section{Discusión}

Ornithobacterium rhinotracheale tiene una variabilidad genética más alta que otros microorganismos (Amonsin et al., 1997;
Vargas et al., 2003), lo cual dificulta la identificación de linajes clonales. Técnicas basadas en tipificación de ADN permiten una mejor caracterización molecular y agrupamiento de acuerdo con las relaciones clonales existentes (Amonsin et al., 1997; Tabatabai et al., 2010). La aplicación de ERIC-PCR para caracterización molecular de las 36 cepas de $O$. rhinotracheale demostró su utilidad en caracterización de la variabilidad genética y la determinación de linajes clonales en el presente estudio.

Réka et al. (2017) y Hassanzadeh et al. (2010) demostraron la existencia de diversificación clonal entre aislados de $O$. rhinotracheale en aves de corral en Hungría e Irán, respectivamente, utilizando similares metodologías (ERIC-PCR), mientras que otros autores lo demostraron en pavos comerciales (Zorman-rojs et al., 2000; Vargas et al., 2003).

Los hallazgos sugieren la presencia de moderada variabilidad genotípica interespecífica e intraespecífica diferenciada dentro de las 36 cepas de $O$. rhinotracheale procedentes de las cinco regiones con al menos 11 perfiles genéticos diferenciados (ERIC-PCR), a diferencia de lo reportado por Koga y Zavaleta (2005), que indican la existencia de un solo perfil genético circulante similar a una cepa control internacional (ATCC $® 51463$ ) utilizando similares técnicas (REP-PCR). Estos resultados sugieren un incremento de la variabilidad genética en esta bacteria en los últimos 13 años.

La mayor variabilidad intraespecífica observada en la región de Lima (7 de 11 perfiles y 5 perfiles únicos) podría explicarse por la mayor concentración de sistemas de producción avícola en esta región; sugiriendo que la presencia de genotipos distintos se debería a la interacción entre diferentes tipos de crianza (producción de carne y huevo comercial) y la posible contaminación por aves silvestres. Macagnan et al. (2006) demostró la presencia de al menos seis genotipos en Brasil 

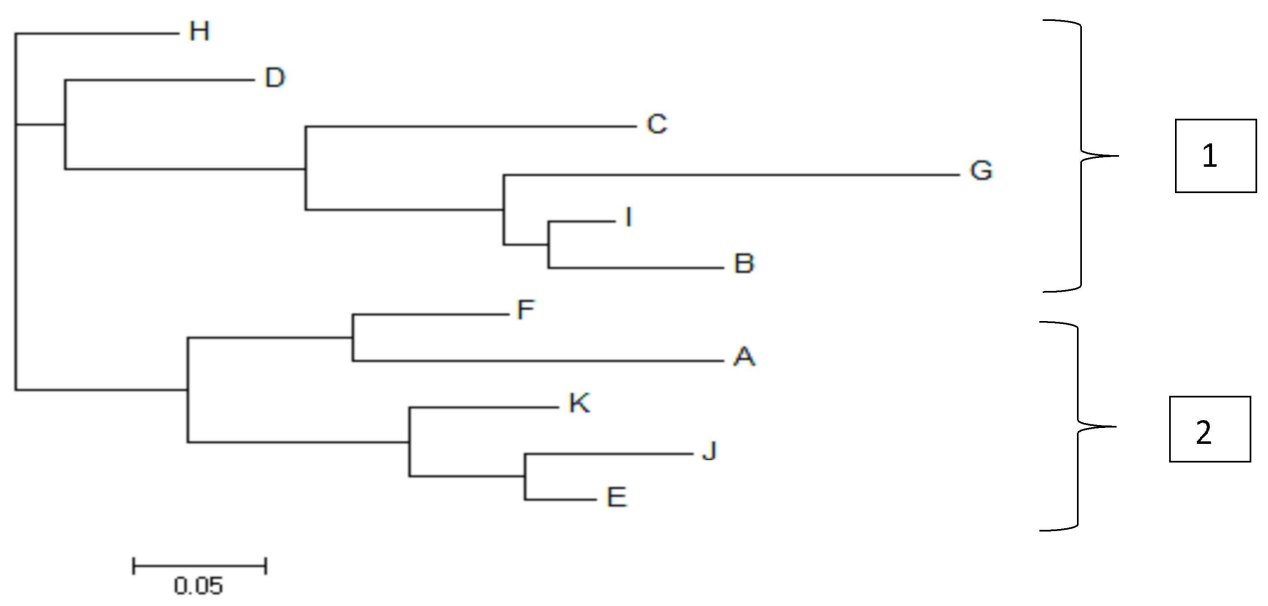

Figura 2. Árbol filogenético mediante método de Neighbor Joining obtenido a partir de una matriz de (Dis) similaridad utilizando el coeficiente de Jaccard y Dice (Mendoza et $a l ., 2014$ ), de DNA fingerprinting (huella genética de ADN) de cepas de $O$. rhinotracheale. Los 11 genotipos presentes en las 36 cepas han sido denominados mediante letras (A-K) para visualizar las relaciones existentes entre los tipos de patrones de bandas

Cuadro 2. Aislados de Ornithobacterium rhinotracheale $(\mathrm{n}=36)$, según el origen de procedencia, y patrones Enterobacterial Repetitive Intergenic Consensus-PCR (ERIC-PCR) diferenciados

\begin{tabular}{cccl}
\hline Patrón ERIC-PCR & $\mathrm{n}$ & $\%$ & Ubicación geográfica \\
\hline $\mathrm{A}$ & 1 & 2.78 & Arequipa \\
$\mathrm{B}$ & 1 & 2.78 & Lima \\
$\mathrm{C}$ & 1 & 2.78 & La Libertad \\
$\mathrm{D}$ & 20 & 55.56 & Lima, La Libertad, Ica y Ucayali \\
$\mathrm{E}$ & 1 & 2.78 & Lima \\
$\mathrm{F}$ & 4 & 11.11 & Lima \\
$\mathrm{G}$ & 2 & 5.56 & La Libertad y Arequipa \\
$\mathrm{H}$ & 1 & 2.78 & La Libertad \\
$\mathrm{I}$ & 3 & 8.33 & Lima y La Libertad \\
$\mathrm{J}$ & 1 & 2.78 & Lima \\
$\mathrm{K}$ & 1 & 2.78 & Lima \\
\hline
\end{tabular}

mediante el single-enzime amplified fragment length polymorphism (SAFLP), sugiriendo que el origen de la variabilidad podría ser contaminación vertical de aves comerciales con aves silvestres (King et al., 2002; Turan y Ak, 2002; Jefferies et al., 2004; Chou et al., 2009).
La baja variabilidad intraespecífica observada en la región de Arequipa debe ser considerada con cuidado, ya que el número de cepas analizados no permitiría explicar la dispersión y presencia de clonas diferenciadas de $O$. rhinotracheale en esta región; sin embargo, los resultados podrían guardar re- 
lación con la presión de selección a la que está sometida la bacteria ante el uso de antimicrobianos y vacunas comerciales (Smith et al., 1993).

Los patrones de resistencia a cinco familias de antibióticos mostraron una elevada multidrogo resistencia (94.4\%), lo cual podría sugerir una fuerte presión de selección con un incremento en la variabilidad genética de $O$. rhinotracheale, debido probablemente a la subdosificación o uso indiscriminado de los antibióticos en las aves de corral (Devriese et al., 1995, 2001; Peña et al., 2016). Resistencia a penicilinas (amoxicilina), tetraciclinas (doxiciclina y tetraciclina) y a quinolonas (enrofloxacina, ciprofloxacina y norfloxacina) variaron entre el 40 y $60 \%$, similar a lo reportado para Brasil y Bélgica (Devriese et al., 1995, 2001; van Veen et al., 2001) y otros países (Zorman et al., 2000; Soriano et al., 2003).

El incremento en la resistencia antimicrobiana podría indicar una constante actividad genética presente en $O$. rhinotracheale observado como un incremento en la variación genética intraespecífica. La aparición de cepas con nuevas características fenotípicas no reportadas previamente (pequeñas colonias variables o la capacidad de hemolítica) (Hsiang-Jung y Chen-Wei, 2006; Tabatabai et al., 2010; Mohammad et al., 2013; Zahra et al., 2013) reforzarían esta hipótesis.

\section{Conclusiones}

- Los resultados indican la presencia de cepas de Ornithobacterium rhinotracheale multidrogo resistentes (94.4\%), con moderada variabilidad genética no organizada a nivel geográfico y de hospedero.

- El patrón genético D fue el más frecuente con un $55.6 \%$ de presencia y distribuido en cuatro de las cinco regiones evaluadas en el país.
- La región Lima concentró la mayor variabilidad genética (7/11 patrones totales) con la presencia de cinco patrones únicos de ERIC-PCR.

\section{Agradecimientos}

El presente trabajo fue financiado y ejecutado en las instalaciones de la empresa Bioservice SRL. Los autores agradecen a los médicos veterinarios Rosario Condori, Stephane Lovón y Manuel Silvera por el apoyo logístico, sugerencias y revisión del manuscrito.

\section{Literatura Citada}

1. Amonsin A, Wellehan J, Li L, Vandamme P, Lindeman C, Edman M, Robinson R, et al. 1997. Molecular epidemiology of Ornithobacterium rhinotracheale. J Clin Microbiol 35: 2894-2898.

2. Chou C, Lin S, Chen C, Tsai H. 2009. Use of random amplified polymorphic DNA analysis and single-enzyme amplified fragment length polymorphism in molecular typing of Ornithobacterium rhinotracheale strains. Avian Dis 53: 108-114. doi: 10.1637/8474092708-Reg.1

3. Devriese L, Hommez, J, Vandamme P, Kersters K, Haesebrouck F. 1995. In vitro antibiotic sensitivity of Ornithobacterium rhinotracheale strains from poultry and wild birds. Vet Rec 137 : 435436. doi: 10.1136/vr.137.17.435

4. Devriese LA., de Herdt P, Haesebrouck F. 2001. Antibiotic sensitivity and resistance in Ornithobacterium rhinotracheale strains from Belgian broiler chickens. Avian Pathol 30: 197-200. doi: 10.1080/030794501200 54596

5. El-Sukhon N, Musa A, Al-Attar M. 2002. Studies on the bacterial etiology of airsacculitis of broilers in Northern and Middle Jordan with special reference to 
Escherichia coli, Ornithobacterium rhinotracheale, and Bordetella avium. Avian Dis 46: 605-612. doi: 10.1637/ $0005-2086(2002)-046[0605$ : SOTBEO]2.0.CO;2

6. Espinoza I, Colas M, Vichi J, Báez M, Martínez S. 2011. Isolation and identification of Ornithobacteriun rhinotracheale from laying hens in farms of La Habana province. Rev Salud Anim 33: 38-43.

7. Ha HJ, Christensen N, Humphrey $S$, Haydon T, Bernardi G, Rawdon T. 2016. The first detection of Ornithobacterium rhinotracheale in New Zealand. Avian Dis 60: 856-859. doi: 10.1637/ 11457-062116-case

8. Hafez H, Sting R. 1999. Investigations on different Ornithobacterium rhinotracheale «ORT» isolates. Avian Dis 43: 1-7.

9. Hafez M, Vandamme P. 2011. Genus XXVI Ornithobacterium. In: Bergey's manual of systematic bacteriology. $2^{\text {nd }}$ ed. New York: Springer. p 250-314.

10. Hassanzadeh M, Karrimi $V$, Fallah $N$, Ashrafi I. 2010. Molecular characterization of Ornithobacterium rhinotracheale isolated from broiler chicken flocks in Iran. Turk J Vet Anim Sci 34: 373-378. doi: 10.3906/vet-0810-19

11. Hsiang-Jung T, Chen-Wei H. 2006. Phenotypic and molecular characterization of isolates of Ornithobacterium rhinotracheale from chickens and pigeons in Taiwan. Avian Dis 50: 502507. doi: 10.1637/7527-031906R.1

12. Hung A, Alvarado A. 2001. Phenotypic and molecular characterization of isolates of Ornithobacterium rhinotracheale from Peru. Avian Dis 45: 999-1005. doi: $10.2307 / 1592880$

13. Jefferies J, Smith A, Clarke S, Dowson C, Mitchell T. 2004. Genetic analysis of diverse disease-causing pneumococci indicates high levels of diversity within serotypes and capsule switching. J Clin Microbiol 42: 5681-5688. doi: 10.1128/ JCM.42.12.5681-5688.2004
14. Joubert P, Higgins $R$, Laperle A, Mikaelian I, Venne D, Silim A. 1999. Isolation of Ornithobacterium rhinotracheale from turkeys in Quebec, Canada. Avian Dis 43: 622-626. doi: $10.2307 / 1592667$

15. King S, Leigh J, Heath P, Luque I, Tarradas C, Dowson C, Whatmore AM. 2002. Development of a multilocus sequence-typing scheme for the pig pathogen Streptococcus suis: identification of virulent clones and potential capsular serotype exchange. J Clin Microbiol 40: 3671-3680. doi: 10.1128/jcm.40.10.3671-3680.2002

16. Koga Y, Zavaleta A. 2005. Intraspecies genetic variability of Ornithobacterium rhinotracheale in commercial birds in Peru. Avian Dis 49: 108-111. doi: 10.1637/ 7235-070804R

17. Macagnan M. 2006. Caracterização fenotípica e genotípica de isolados de Ornithobacterium rhinotracheale do Brasil. Tesis de Maestría. Porto Alegre, Brasil: Universidad Federal do Rio Grande do Soul. $51 \mathrm{p}$.

18. Mendoza K, Zavaleta A, Koga Y, Rodríguez J, Alvarado A, Tinoco $R$. 2014. Variabilidad genética de cepas de Gallibacterium anatis aisladas de aves comerciales del Perú con infecciones respiratorias. Rev Inv Vet Perú 25: 233 244. doi: 10.15381/rivep.v25i2.8496

19. Mohammad Z, Miro F, Rashad A, Jinhua Y, Bo H, Jingliang S. 2013. Isolation and characterization of smallcolony variants of Ornithobacterium rhinotracheale. J Clin Microbiol 51: 3228-3236. doi: 10.1128/JCM.01337-13

20. [NCCLS] Clinical and Laboratory Standard Institute. 2017. Performance standards for antimicrobial disk and dilution susceptibility test for bacteria isolated from animals. $2^{\text {nd }}$ ed NCCLS M31-A2. Vol 19(1): 1-87.

21. Peña E, Vega V, Morales V, Trujillo H, Talavera M, Soriano E. 2016. Serotyping, genotyping, and antimicrobial susceptibility of Ornithobacterium rhinotracheale isolates from Mexico. 
Avian Dis 60: 669-672. doi: 10.1637/ 11333-112515-ResNote.1

22. Réka S, Enik̃̃ W, László M, Csaba N, Éva G, Ákos T, Tibor M. 2017. Characterization of Ornithobacterium rhinotracheale field isolates from Hungary. Avian Pathol 46: 506-514. doi: 10.1080/03079457.2017.1321104

23. Sakai E, Tokuyma Y, Nonaka F, Ohishi S, Ishikawa Y, Tanaka M, Taneno A. 2000. Ornithobacterium rhinotracheale infection in Japan: preliminary investigations. Vet Rec 146: 502-504. doi: 10.1136/vr.146.17.502

24. Schlüter M, Harris SA. 2006. Analysis of multilocus fingerprinting data sets containing missing data. Molecular. Mol Ecol Notes 6: 569-572. doi: 10.1111/ j.1471-8286.2006.01225.x

25. Smith M, Smith H, O'Rourke M, Spratt G. 1993. How clonal are bacteria? P Natl Acad Sci USA 90: 4384-4388. doi: $10.1073 /$ pnas.90.10.4384

26. Soriano V, Longinos M, Navarrete P, Fernández R. 2002. Identification and characterization of Ornithobacterium rhinotracheale isolates from Mexico. Avian Dis 46: 686-690. doi: 10.1637/00052086(2002)046-[0686:IACOOR]2.0.CO;2

27. Soriano VE, Vera NA, Salado CR, Fernández RP, Blackall PJ. 2003. In vitro susceptibility of Ornithobacterium rhinotracheale to several antimicrobial drugs. Avian Dis 47: 476-480. doi: 10.1637/ 0005-2086(2003)-047[0476:IVSOOR]2.0.CO;2

28. Tabatabai B, Zimmerli K, Zehr S, Briggs E, Tatum M. 2010. Ornithobacterium rhinotracheale North American field isolates express a hemolysinlike protein. Avian Dis 54: 994-1001. doi: 10.1637/9070-091409-Reg.1

29. Tamura K, Stecher G, Peterson D, Filip A, Kumar S. 2013. MEGA6: Molecular evolutionary genetics analysis version 6.0. Mol Biol Evol 30: 2725-2729. doi: $10.1093 / \mathrm{molbev} / \mathrm{mst} 197$

30. Turan N, Ak S. 2002. Investigation of the presence of Ornithobacterium rhinotracheale in chickens in Turkey and determination of the seroprevalence of the infection using the enzyme-linked immunosorbent assay. Avian Dis 46: 442446. doi: 10.1637/0005-2086(2002)046[0442:IOTPOO]2.0.CO;2

31. van Empel C, Hafez M. 1999. Ornithobacterium rhinotracheale: a review. Avian Pathol 28: 217-227. doi: 10.1080/03079459994704

32. van Veen L, Hartman E, Fabri T. 2001. In vitro antibiotic sensitivity of strains of Ornithobacterium rhinotracheale isolated in The Netherlands between 1996 and 1999. Vet Rec 149: 611-613. doi: 10.1136/vr.149.20.611

33. Vandamme P, Segers P, Vancaneyt M, van Hover K, Mutters R, Hommez J, Dewirst F, et al. 1994. Description of Ornithobacterium rhinotracheale gen. Nov. sp. Nov. isolated from the avian respiratory tract. Int J Syst Evol Micr 44: 24-37. doi: 10.1099/00207713-44-1-24

34. Vargas S, Garduño L, Rosas F. 2003. Isolation and characterization of Ornithobacteriun rhinotracheale from respiratory diseased turkeys. Vet México 34: 283-288.

35. Zahra M, Ferreri M, Alkasir R, Yin J, Han B, Su J. 2013. Isolation and characterization of small-colony variants of Ornithobacterium rhinotracheale. J Clin Microbiol 51: 3228-3236. doi: 10.1128/JCM.01337-13

36. Zorman-Rojs O, Zdovc I, Bencina D, Mrzel I. 2000. Infection of turkeys with Ornithobacterium rhinotracheale and Mycoplasma synoviae. Avian Dis 44: 1017-1022. doi: 10.2307/1593082 